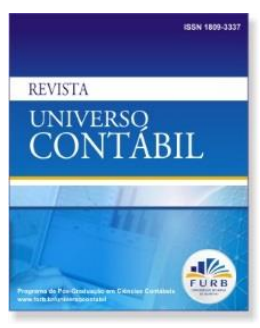

Revista Universo Contábil, ISSN 1809-3337

Blumenau, v. 9, n. 2, p. 45-62, abr./jun., 2013

doi:10.4270/ruc.2013212

Disponível em www.furb.br/universocontabil

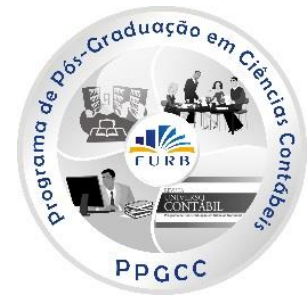

IMPACTO DO REGISTRO DE OFERTAS PÚBLICAS SUBSEQUENTES NOS RETORNOS DE AÇÕES DE EMPRESAS LISTADAS NO SEGMENTO BOVESPA ${ }^{1}$

\title{
IMPACT OF THE REGISTER OF SEASONED OFFERINGS IN THE RETURNS OF SHARES OF THE COMPANIES LISTED IN THE BOVESPA SEGMENT
}

\section{IMPACTO DE LAS OFERTAS DE LOS REGISTROS PÚBLICOS EN LAS DEVOLUCIONES DE ACCIONES POSTERIORES DE LAS EMPRESAS QUE FIGURAN EN EL SEGMENTO BOVESPA}

Nara Grossi Teixeira Guimarães

Bacharel em Ciências Contábeis pela UFVJM Endereço: Rua do Cruzeiro, 01 - Jardim São Paulo CEP: 39803-371 - Teófilo Otoni - MG

E-mail: narahgrossi@yahoo.com.br Telefone: (33) 8808-1110

Oscar Neto de Almeida Bispo

Mestre em Ciências Contábeis pela UFMG Professor do Curso de Ciências Contábeis da UFVJM

Rua do Cruzeiro, 01 - Jardim São Paulo CEP: 39803-371 - Teófilo Otoni - MG E-mail: oscar.almeida@ufvjm.edu.br Telefone: (33) 3522-6037

Marcos Valério Martins Soares Especialista em Ciências Contábeis pela Faculdade Doctum Professor do Curso de Ciências Contábeis da UFVJM Rua do Cruzeiro, 01 - Jardim São Paulo CEP: 39803-371 - Teófilo Otoni - MG E-mail:marcos.valerio@ufvjm.edu.br Telefone: (33) 3522-6037

\section{Vagner Antônio Marques}

Doutorando em Administração pela UFMG Professor da PUCMINAS Av. Afonso Vaz de Melo, 1200 - Barreiro de Baixo

CEP: 30640-070 - Belo Horizonte - MG

E-mail:vmarques@pucminas.br Telefone: (31) 3319-4444

\footnotetext{
${ }^{1}$ Artigo recebido em 12.04.2012. Revisado por pares em 24.10.2012. Reformulado em 24.05.2013. Recomendado para publicação em 22.06.13 por Carlos Eduardo Facin Lavarda. Publicado em 30.06.2013. Organização responsável pelo periódico: FURB.
} 
Nara Grossi Teixeira Guimarães - Oscar Neto de Almeida Bispo - Marcos Valério Martins Soares Vagner Antônio Marques

\section{RESUMO}

A presente pesquisa teve como objetivo geral analisar os impactos das ofertas públicas subsequentes (Seasoned Equity Offerings - SEO) nos retornos de ações de empresas listadas no segmento Bovespa, que fizeram emissões entre os anos de 2001 a 2010. Especificamente, pretende-se identificar a reação do mercado de capitais frente à emissão de novas ações, verificar os retornos normais dos títulos e verificar se há retornos anormais quando da emissão subsequente de ações. Para analisar tais reflexos aplicou-se a metodologia de estudo de evento para o evento de registro da emissão subsequente de ações na Comissão de Valores Mobiliários. O estudo foi aplicado em uma amostra de 27 emissões de 20 companhias distintas, de variados setores, procurando-se determinar como o mercado reagiu antes, durante e depois da data do registro na Comissão de Valores Mobiliários. Não foram encontrados retornos anormais estatisticamente significativos, pois todos os p-valores são maiores que o nível de significância utilizado na pesquisa. Portanto, não se rejeitou a hipótese nula de que os retornos anormais médios são iguais a zero. Os resultados também apontaram que não ocorreram retornos anormais estatisticamente significativos na janela de comparação, confirmando a ausência de reflexos do evento nos retornos das ações das empresas que realizaram o registro de Ofertas Públicas Subsequentes de Ações.

Palavras-chave: Seasoned Equity Offering. Eficiência de Mercado. Estudo de Eventos.

\section{ABSTRACT}

The present research had as objective generality to analyze the impacts of the Seasoned Public Offerings - SEO in the returns of shares of companies listed in the Bovespa segment, who had made emissions between the years of 2001 the 2010. Specifically, it is intended to identify the reaction of the stock market front to the emission of new shares, to check the normal returns of the titles and to verify if it has abnormal returns when of the Seasoned Public Offerings of Shares. To analyze such consequences it was applied methodology of study of event for the event of register of the Seasoned Public Offerings of Shares in the Commission of Movable Values. The study company was applied in a sample of 27 distinct 20 emissions of the varied sectors, looking itself to determine as the market reacted before, during and after the date of the register in the Commission of Movable Values. The determination model was the model of the return adjusted to the risk and the market. The found empirical results had statistical pointed significant abnormal returns in first, fourth and fifth day after the register in the Commission of Movable Values. There were no statistically significant abnormal returns, because all p-values are greater than the significance level used in the research. So do not reject the null hypothesis that the average abnormal returns are equal to zero. The results also showed that there were no statistically significant abnormal returns in the window of comparison, confirming the absence of reflexes in the event stock returns of companies that held the record Subsequent Public Offerings of Shares.

Keywords: Seasoned Equity Offering. Market Efficiency. Events Study.

\section{RESUMEN}

La presente investigación tuvo como objetivo general analizar los impactos de las ofertas de los registros públicos (Seasoned Equity Offerings - SEO) en las devoluciones de acciones posteriores de las empresas que figuran en el segmento Bovespa, que hicieron emisiones entre los años de 2001 a 2010. Específicamente tiene como objetivo identificar la reacción de los mercados de capital frente a la emisión de nuevas acciones, comprobar los rendimientos de los títulos normales y comprobar si hay rendimientos anormales al emitir las acciones posteriores. Para analizar estas reflexiones se aplicó la metodología de caso de estudio para el registro de 
eventos de la emisión de acciones posteriores en la Comisión de Valores Mobiliarios. El estudio fue aplicado en una muestra de 27 emisiones de 20 empresas diferentes de varios sectores, buscando determinar cómo el mercado reaccionó antes, durante y después de la fecha del registro en la Comisión de Valores Mobiliarios. El modelo fue el modelo para la determinación de la rentabilidad ajustada al riesgo y al mercado. Los resultados empíricos encontrados señalaron retornos anormales estadísticamente significativos en el segundo, tercer y cuarto día después del registro de la Comisión de Valores Mobiliarios. No hubo retornos anormales estadisticamente significativos, ya que todos los p-valores son mayores que el nivel de significación utilizado en la investigación. Así que no se rechaza la hipótesis nula de que las rentabilidades medias anormales son iguales a cero. Los resultados también mostraron que no había retornos anormales estadísticamente significativos en la ventana de comparación, lo que confirma la ausencia de reflejos en los rendimientos de las acciones de eventos de empresas que tenía el récord de la Oferta Pública de Acciones posteriores.

Palabras clave: Seasoned Equity Offering. Eficiencia de Mercado. Estudio de Eventos.

\section{INTRODUÇÃO}

Os estudos que envolvem as formas de financiamento das empresas têm despertado interesse em diversas pesquisas. Tais interesses decorrem da preocupação existente com o mix de instrumentos utilizados para financiá-las, surgindo com isso a inquietação voltada à estrutura de capital mais adequada para a manutenção de suas operações. Nesse contexto, vislumbrando maior acesso a financiamento de projetos de expansão e investimento, algumas empresas recorrem ao mercado acionário e optam por uma estrutura de capital que contemple o acréscimo de recursos adicionais por meio da subscrição de ações. Para a BM\&FBOVESPA (2012) o mercado acionário brasileiro tem adquirido cada vez mais relevância no financiamento das empresas, constituindo uma fonte alternativa para captação de recursos financeiros visando a concretização das oportunidades de crescimento e desenvolvimento.

Pode-se ressaltar que no decorrer das atividades das empresas sempre haverá oportunidades de crescimento e desenvolvimento, e a necessidade de aumento de capital poderá ocasionar o retorno ao mercado acionário para a venda de novas ações, por meio de Ofertas Públicas Subseqüentes (Seasoned Equity Offering - SEO), acarretando na aquisição de novos sócios e consequentemente novo financiamento para suas atividades. Destaca-se que, ao obterem recursos financeiros, seja por meio da emissão inicial, ou emissão subsequente de ações, as empresas compartilham os riscos com os investidores e não há a obrigação da restituição integral destes recursos, visto que os resultados futuros dependem das probabilidades de ocorrência de preços e retornos do investimento, podendo estes não ocorrer como esperado.

A análise da divulgação de eventos dessa magnitude torna-se relevante para o mercado de capitais e consequentes avaliações das perspectivas de rentabilidade futura das ações negociadas em bolsa de valores. Ademais, é importante ficar atento aos acontecimentos do mercado e aos fatos relevantes divulgados pelas empresas. Uma forma de analisar as informações relevantes disponibilizadas ao mercado de capitais é o Estudo de Eventos. Para Fama (1991) o Estudo de Eventos consiste na análise do efeito das informações relevantes específicas para cada empresa sobre os preços de suas ações negociadas em bolsas de valores.

Camargos e Barbosa (2003b, p. 1) complementam que o Estudo de Eventos "pode ser realizado em vários eventos importantes que impactam as expectativas dos investidores e, consequentemente, os preços dos títulos". Pode-se destacar entre esses eventos: o anúncio da deliberação pela subscrição de ações em bolsas de valores, o anúncio de registro de ofertas públicas de aquisições de ações, o registro de emissões de debêntures, a evidenciação contábil 
Nara Grossi Teixeira Guimarães - Oscar Neto de Almeida Bispo - Marcos Valério Martins Soares Vagner Antônio Marques

de lucros/prejuízos nas demonstrações de resultados publicadas, pagamento de dividendos, entre outros.

Na concepção de Campbell, Lo e MacKinlay (1997), um Estudo de Eventos avalia o impacto de um evento específico no valor da empresa e sua utilidade vem do fato que, admitindo a eficiência do mercado, o efeito de um evento em particular será refletido imediatamente nos preços dos ativos, nesse caso o preço das ações. Assim, justifica-se a realização do Estudo de Eventos em dias próximos ao registro de Ofertas Públicas Subsequentes de Ações para medir o impacto econômico nos retornos das ações, avaliando se ocorreram retornos anormais, ou seja, apresentaram resultados diferentes daqueles que eram esperados. Uma pesquisa que investigue os impactos do retorno, quando da emissão de novas ações, revela-se importante na contribuição para o mercado de capitais, pois reforça a discussão em torno da reação do mercado frente a estas emissões e frente a uma informação específica.

Observa-se, então, a necessidade de pesquisas que visam analisar as reações do mercado de capitais frente às emissões de ações, mais especificamente as Ofertas Públicas Subsequentes. O risco, o retorno e outros reflexos advindos desse tipo de oferta podem provocar impactos substanciais para este mercado. Diante disso, o objetivo geral da pesquisa é analisar os impactos das Ofertas Públicas Subsequentes nos retornos de ações de empresas listadas no segmento Bovespa, que fizeram emissões entre os anos de 2001 a 2010. Especificamente, pretende-se identificar a reação do mercado de capitais frente à emissão de novas ações, verificar os retornos normais dos títulos e verificar se há retornos anormais quando da emissão subsequente de ações.

Para complementar, a pesquisa está estruturada da seguinte forma: no tópico seguinte tem-se a discussão teórica do trabalho; em seguida são discorridos os procedimentos metodológicos para consecução dos resultados; no tópico 4 tem-se a exposição dos resultados encontrados pelo estudo; e, por fim, são abordas as considerações finais da pesquisa.

\section{REFERENCIAL TEÓRICO}

\subsection{Ofertas Públicas Subseqüentes de Ações (Seasoned Equity Offering - SEO)}

Conforme Dias (2007), um dos temas centrais da teoria de finanças corporativas diz respeito à estrutura de capital das empresas, ou seja, a forma como as empresas utilizam capital, se próprio ou de terceiros. O capital próprio corresponde aos recursos oriundos dos sócios ou acionistas, enquanto o de terceiros compreende os recursos financeiros obtidos de terceiros por meio de dívidas.

Para Bispo (2010), a política de financiamento adotada pela empresa é que vai indicar o mix de instrumentos utilizados para financiá-la. Entretanto, de acordo com Brigham e Ehrhardt (2006), as empresas devem buscar estabelecer uma estrutura de capital ótima. Essa estrutura pode sofrer mudanças ao longo do tempo, conforme as condições financeiras da empresa e do mercado se modifiquem. Bispo (2010) ressalta ainda que, deve ficar evidente que a estrutura de capital ótima deve buscar um equilíbrio entre risco e retorno naquelas escolhas colocadas em prática, no intuito de maximizar o valor da empresa. De tal modo, os impactos das decisões de financiamento sobre o valor da empresa vão apontar os determinantes da escolha da estrutura de capital.

De acordo com a teoria do pecking order (hierarquização das fontes de financiamento), a escolha ótima da estrutura de capital das empresas priorizará o financiamento por lucros retidos (autofinanciamento), seguido por dívidas e, em último caso, a emissão de ações (MYERS, 1984). Porém, dentre essas escolhas, o autofinanciamento nem sempre é suficiente, sendo necessária a incorporação de capital externo para financiar os projetos de investimentos. Assim, caso uma empresa já tenha seu capital aberto, uma das opções para uma nova incorporação de capital seria a Oferta Pública Subsequente de Ações. 
Conforme ressaltado por Iquiapaza et al. (2009) as Ofertas Públicas Subsequentes de Ações podem ser direcionados aos investidores das seguintes formas: (i) forma restrita, quando somente permite-se a subscrição pelos atuais acionistas; e, (ii) forma ampla, em que se possibilita a subscrição por quaisquer investidores, chamados lançamentos públicos de ações. De acordo com Berk e DeMarzo (2009) a primeira hipótese refere-se ao fato de que a empresa oferece as novas ações apenas para os atuais acionistas. Estas protegem os acionistas existentes contra a subprecificação. Já a segunda hipótese refere-se ao fato de que a empresa oferece as novas ações aos diversos investidores no mercado de capitais. Tais hipóteses devem estar evidentes nos prospectos (relatórios da emissão) da oferta subseqüente.

Apesar das empresas publicarem prospectos com os dados referentes às SEOs, Teoh, Wong e Rao (1998) encontraram evidências de que os investidores, ingenuamente, exageram em suas análises quanto aos resultados das empresas em momentos que antecedem uma SEO, ignorando informações relevantes contidas nas demonstrações financeiras antes da oferta. Tais análises podem influenciar os preços e impactar negativamente nos retornos das ações em períodos próximos às emissões subsequentes. Hess e Frost (1982, apud IQUIAPAZA et al., 2009, p. 20) já haviam investigado os movimentos de preços nas imediações da data de emissão de uma SEO, comparando três hipóteses distintas: (i) a visão de que uma nova emissão causa uma queda permanente de preço; (ii) a visão de que a queda nos preços é temporária, ocorrendo durante o período de distribuição; e, (iii) a hipótese de mercado eficiente, em que o efeito sobre os preços é nulo. A conclusão daqueles dois autores foi de rejeitar as duas primeiras hipóteses em detrimento da última, a de mercado eficiente.

Outros estudos (LOUGHRAN; RITTER, 1997, RANGAN, 1998; DENIS; SARIN, 2001) também verificaram que, em média, o mercado recebe a notícia de uma SEO com uma queda no preço das ações e, consequentemente, no valor de mercado da empresa, o que de certa forma impacta negativamente os retornos das ações.

\subsection{Hipótese da Eficiência de Mercado - HEM}

Camargos e Barbosa (2003a, p. 41) ressaltam que "a HEM, que é um dos pilares da Moderna Teoria de Finanças, está baseada na premissa de que os preços dos títulos refletem instantaneamente todas as informações relevantes disponíveis no mercado". Os autores ainda salientam que os mercados de capitais informacionalmente eficientes proporcionam economia de tempo e de recursos que seriam gastos na análise de informações, uma vez que estas já estão refletidas nos preços. Para Hendriksen e Breda (1999), pode-se afirmar que um mercado é eficiente em relação a uma dada informação, quando fica impossível de se obter lucros extraordinários usando tal informação para negociar.

Para Assaf Neto (2007, p. 205) "toda nova informação relevante trazida ao mercado tido como eficiente tem o poder de promover alterações nos valores dos ativos negociados, modificando seus livres preços de negociação e resultados de análises". Hendriksen e Breda (1999, p. 117) complementam que:

\footnotetext{
[...] as condições suficientes para a existência do mercado eficiente são: (i) não há custos de transação na negociação de títulos; (ii) toda informação disponível está igualmente disponível a todos os participantes do mercado, sem qualquer custo; e, (iii) todos os participantes do mercado possuem expectativas homogêneas em relação às implicações da informação disponível.
}

Observa-se que o mercado de capitais é considerado eficiente, mais precisamente em relação a um conjunto particular de informações, se e somente se os preços dos ativos negociados nesse mercado possuírem comportamento como se todos os participantes do mercado observassem os sinais produzidos pelo sistema de informação. Portanto, entende-se que as diversas versões do conceito de eficiência possuem uma única divergência: os diferentes níveis de informação que devem refletir os preços do mercado. 
Nara Grossi Teixeira Guimarães - Oscar Neto de Almeida Bispo - Marcos Valério Martins Soares Vagner Antônio Marques

Conforme descrito em Camargos e Barbosa (2003a, p. 45-48) há três níveis de eficiência existentes:

(i) A HEM, na sua forma fraca, indica que o mercado incorpora completamente as informações sobre os preços passados dos títulos. Ou seja, retornos anormais não podem ser obtidos baseados nas expectativas de que os preços passados são bons sinalizadores dos preços futuros.

(ii) A HEM, na sua forma semiforte, indica que os preços refletem não apenas o histórico do comportamento de preços, como também todas as informações disponíveis publicamente (demonstrativos financeiros e outras publicações periódicas e não-periódicas). Nenhum investidor consegue obter retornos extraordinários baseado em informações públicas, pois os preços se ajustam rapidamente às novas informações divulgadas.

(iii) A HEM, na sua forma forte, indica que os preços refletem todas as informações existentes no mercado (históricas, públicas e privadas). Assim, um insider, de posse de informações privadas, não conseguiria auferir retornos extraordinários, pois o ajuste dos preços no mercado é instantâneo.

Inerente às Hipóteses de Eficiência de Mercado, Santos et al. (2007) explicam que problemas provocados por diferenças na informação podem acarretar em informações assimétricas. Tais problemas surgem quando compradores e vendedores não estão corretamente informados sobre a qualidade dos bens que são vendidos no mercado, ambos têm diferentes informações sobre os bens comercializados.

Até que a informação esteja disponível ou incorporada no preço das ações, cria-se um problema de seleção adversa impondo risco informacional aos participantes de mercado não informados. Dado que o lucro do investidor informado é igual à perda do investidor não informado, este tenderá a se proteger contra o risco informacional demandando maiores retornos ex-ante. Ao permitir esta transferência de riqueza entre os investidores no mercado secundário, a assimetria informacional faz com que o custo de capital da empresa aumente exante, quando da emissão de ações ou títulos de dívida no mercado primário (BRESSAN et al., 2007, p. 225).

Levando-se em consideração a evidenciação simétrica de informações das empresas ao mercado, pode-se melhorar a previsibilidade futura dos retornos a serem apresentados pelas ações negociadas em bolsas de valores. De acordo com Ferreira (2008) os investimentos possuem graus diferentes em relação ao risco associado à sua imprevisibilidade futura, podendo ser classificados pelo nível de segurança no investimento, que é inversamente proporcional ao risco. A segurança de uma aplicação está ligada a menores possibilidades de perdas, porém, apresentam menos retorno ao investidor, ao mesmo tempo em que aplicações com maior risco oferecem maiores retornos. Portanto, classificam-se os retornos em: retornos esperados e retornos anormais. $\mathrm{O}$ primeiro, também chamado de retornos normais, são expectativas futuras de ganhos com ativos com risco. O segundo, ao contrário do retorno esperado, é incerto e sem previsão de advir.

\section{PROCEDIMENTOS METODOLÓGICOS}

\subsection{Classificação da pesquisa}

Nesta pesquisa, utilizou-se uma abordagem positiva, empregando como técnica de investigação o estudo descritivo. Conforme Gil (2007), esse tipo de estudo tem como principal objetivo descrever características de determinada população, ou fenômeno, ou estabelecer relações entre as variáveis.

O tratamento dos dados foi realizado com o uso de técnicas estatísticas e, geralmente, a validação do resultado foi apoiada nos níveis estatísticos de significância. Na abordagem positiva, considera-se que possam existir relações de causa e efeito entre eventos, entendidas 
como relações entre variáveis dependentes e independentes. No caráter epistemológico, quanto aos objetivos, esta pesquisa pode ser classificada como pesquisa explicativa, ex-post-facto.

\subsection{Procedimentos de coleta de dados}

Para consecução dos resultados o estudo ampara-se na utilização dos pressupostos teórico/práticos do Estudo de Eventos relacionados à divulgação de informações relativas ao registro de Ofertas Públicas Subsequentes de Ações de empresas de capital aberto, listadas no segmento Bovespa. Os dados foram extraídos do site da Comissão de Valores Mobiliários CVM, compreendendo as Ofertas públicas Subsequentes de Ações realizadas entre os anos de 2001 a 2010.

Como delimitações da amostra de estudo foram utilizados os dados de eventos relacionados às empresas que participaram da carteira teórica do Índice Bovespa - Ibovespa no período de 2001 a 2010. Esse critério foi estabelecido devido aos pressupostos teóricos para a utilização dos conceitos relacionados ao Modelo de Mercado de Estudo de Eventos procedimento empírico utilizado nesta pesquisa. O Modelo de Mercado relaciona linearmente o retorno das ações com o retorno de mercado, indicado pelo retorno do Ibovespa.

A utilização do Ibovespa deve-se ao fato deste ser um indicador do desempenho médio das cotações do mercado de ações brasileiro, sendo considerado um indicador que avalia o retorno total de suas ações componentes. As ações integrantes da carteira teórica do Ibovespa respondem por mais de $80 \%$ do número de negócios e do volume financeiro verificado no mercado à vista, apresentando alta liquidez em transações. Ademais, para a utilização dos procedimentos empíricos baseados no Modelo de Mercado, devem ser utilizados dados que proporcione o cálculo dos retornos normais e anormais das ações. No caso de ações consideradas de baixa liquidez, ou seja, número restrito de negócios com a ação da empresa nos pregões da BM\&FBovespa, abre-se a possibilidade de inviabilizar os cálculos dos retornos normais e anormais, visto a inexistência de dados suficientes para a realização dos cálculos. Quanto maior a liquidez nas negociações, maior a possibilidade de agregar valor aos resultados sem necessitar utilizar procedimentos subjetivos para solucionar possíveis problemas de negociações infrequentes.

Assim, para que os resultados da pesquisa ilustrassem melhor o mercado, permitindo melhor análise dos dados, não compõem a amostra de estudo (Tabela 1) as empresas que demonstraram problemas de liquidez nas negociações de suas ações, aquelas que apresentaram períodos extensos sem negociações na BM\&FBovespa (superiores a 20 pregões subsequentes). É o caso da empresa Eletropaulo, que emitiu ações subsequentes no ano de 2006, mas verificouse a ausência de negociações na bolsa durante um período superior a 20 pregões subsequentes.

Conforme mencionado, utilizou-se a técnica do Estudo de Eventos para analisar empiricamente o comportamento dos retornos das ações. $\mathrm{O}$ evento estudado foi definido como o dia do registro da Oferta Pública Subsequentes de Ações pelas empresas, ou seja, quando a empresa realizou nova distribuição de ações no mercado. A data foi definida como aquela do registro na CVM, sendo composta por 38 emissões subsequentes (Tabela 1). Algumas empresas apresentam duas ou mais datas, pois realizaram mais de uma distribuição subsequente de ações. 
Nara Grossi Teixeira Guimarães - Oscar Neto de Almeida Bispo - Marcos Valério Martins Soares Vagner Antônio Marques

Tabela 1 - Registro na CVM (38 eventos - data 0)

\begin{tabular}{lclc}
\hline \multicolumn{1}{c}{ Empresa } & Registro na CVM & \multicolumn{1}{c}{ Empresa } & Registro na CVM \\
\hline Ambev & $17 / 09 / 10$ & Gafisa & $24 / 03 / 10$ \\
Banco Bradesco & $09 / 03 / 01$ & Gerdau & $08 / 12 / 04$ \\
Banco Bradesco & $14 / 04 / 08$ & Gerdau & $25 / 04 / 08$ \\
Banco do Brasil & $27 / 06 / 06$ & Gol & $09 / 10 / 09$ \\
Banco do Brasil & $14 / 12 / 07$ & JBS & $28 / 04 / 10$ \\
Banco do Brasil & $01 / 07 / 10$ & Light & $14 / 07 / 09$ \\
Bradespar & $20 / 02 / 01$ & MMX & $20 / 12 / 10$ \\
Bradespar & $15 / 12 / 04$ & Natura & $31 / 07 / 09$ \\
Bradespar & $15 / 09 / 05$ & Perdigão & $27 / 10 / 06$ \\
Braskem & $23 / 09 / 04$ & Perdigão & $13 / 12 / 07$ \\
CESP & $02 / 12 / 05$ & Petrobrás & $19 / 07 / 01$ \\
CESP & $28 / 07 / 06$ & Petrobras & $24 / 09 / 10$ \\
CSN & $28 / 03 / 03$ & RedeCard & $25 / 03 / 09$ \\
Cyrela & $28 / 10 / 09$ & Rossi Residencial & $02 / 10 / 09$ \\
Eletrobrás & $20 / 04 / 01$ & Sabesp & $09 / 05 / 02$ \\
Eletrobrás & $01 / 11 / 01$ & Sabesp & $29 / 10 / 04$ \\
Eletropaulo & $24 / 09 / 01$ & Usiminas & $26 / 04 / 07$ \\
Embraer & $13 / 06 / 01$ & Vale do Rio Doce & $21 / 03 / 02$ \\
Embraer & $07 / 02 / 07$ & Vale do Rio Doce & $17 / 07 / 08$ \\
\hline
\end{tabular}

Fonte: elaborada pelos autores.

Para dar andamento ao estudo foram estabelecidas as janelas de evento, estimação e comparação. Por meio dessa metodologia, calculam-se os retornos anormais para determinar um padrão para a identificação das anormalidades dos retornos das ações. Determinou-se para esta pesquisa a janela de eventos com cinco pregões anteriores e subsequentes em torno da data zero, totalizando um período de 11 pregões. Os pregões anteriores à data zero visam analisar se há indícios de aproveitamento de informações privilegiadas, já os pregões posteriores à data zero visam à observação do mercado frente à ocorrência do evento.

Além da janela de eventos, definiu-se a janela de estimação e a janela de comparação. A janela de estimação foi determinada para o cálculo dos retornos normais (ou esperados), sendo composta por 35 pregões anteriores à janela de eventos. Já a janela de comparação irá verificar se os retornos continuam, ou não, a apresentar comportamento anormal após a janela de evento. De forma proporcional à janela de estimação, esta foi composta por 35 pregões posteriores à janela de eventos. Justifica-se a utilização de 35 pregões, tanto na janela de estimação quanto na janela de comparação, no intuito de não sobrepor outros eventos relacionados ao registro das Ofertas Públicas Subsequentes. Observou-se que algumas empresas apresentaram, entre 35 e 90 pregões anteriores à janela de evento, eventos deliberativos diretamente relacionados às ofertas subsequentes. Dessa forma, ao expandir a janela de estimação estes eventos, definidos como Reunião do Conselho de Administração e Assembleia Geral Extraordinária, estariam sobrepondo a janela e comprometendo as análises. Complementa-se que estes eventos deliberativos ocorrem em momentos distintos ao evento de registro, quando efetivamente acontece a emissão das ações aos investidores.

\subsection{Procedimentos Estatísticos}

\subsubsection{Estimativa dos Retornos Normais e Anormais}

Camargos e Barbosa (2003a) afirmam que a metodologia de estudo de eventos, através de um método de cálculo de retornos normais, resulta num padrão para a identificação das anormalidades dos retornos dos títulos, e que esse padrão estima os retornos dos títulos que 
seriam esperados caso o evento não houvesse ocorrido. Os retornos normais (ou esperados) serão estimados a partir do Ibovespa e dos retornos observados das ações na janela de estimação, diferentemente dos retornos anormais, calculados nas janelas de evento e comparação.

Os retornos das ações $\left(R_{i, t}\right)$ e do Ibovespa serão calculados conforme as cotações diárias coletadas, dados pelo $l n$ da relação entre o preço da ação no período $t\left(P_{i, t}\right)$ e o preço da ação no período $t-1\left(P_{i, t-1}\right)$ :

$$
R_{i, t}=\ln \left(\frac{P_{i, t}}{P_{i, t-1}}\right)
$$

Por apresentar uma distribuição mais próxima da distribuição normal, Takamatsu, Lamounier e Colauto (2008), consideram o modelo logarítmico como sendo o mais apropriado para análises empíricas e testes estatísticos.

Para determinação dos retornos anormais recorda-se que foi utilizado o Modelo de Mercado. Trata-se de um modelo estatístico que assume os pressupostos de que os retornos dos ativos são independentes e identicamente distribuídos ao longo do tempo. Tal modelo admite que os retornos anormais das ações sejam observados pela divergência dos retornos individuais $\left(R_{i, t}\right)$ efetivamente ocorridos em relação ao retorno do portfólio do mercado $\left(R_{m, t}\right)$, calculados por um modelo de fator simples. Os coeficientes do modelo $\left(\alpha_{i}\right.$ e $\left.\beta_{i}\right)$ são estimados a partir do método dos mínimos quadrados ordinários (TAKAMATSU; LAMOUNIER; COLAUTO, 2008). Assim, os retornos normais seriam obtidos da seguinte forma:

$$
R_{i, t}=\alpha_{i}+\beta_{i} R_{m, t}+\varepsilon_{i, t}
$$

A partir da equação anterior supõe-se que desvios nos comportamentos dos retornos são captados pelo erro da estimativa $\left(\varepsilon_{i, t}\right)$, o que resultará em retornos anormais $\left(A R_{i, t}\right)$. Portanto, tem-se que:

$$
A R_{i, t}=\varepsilon_{i, t}=R_{i, t}-\alpha_{i}-\beta_{i} R_{m, t}
$$

Camargos e Barbosa (2003a) ressaltam a dificuldade que pode existir em determinar a exata data em que o mercado recebeu as informações do evento e assim, considera-se a acumulação dos retornos anormais (CAR - Cumulative Abnormal Return) nos dias próximos ao evento estudado. Para os autores a acumulação dos retornos anormais pode levar à obtenção de uma melhor análise dos resultados do evento, pois fornecem informações da trajetória seguida pelos retornos em um período de tempo mais significativo para fins de análise. A técnica de retornos anormais acumulados (CAR - Cumulative Abnormal Return) pode ser expressa pela seguinte fórmula:

$$
\operatorname{CAR}_{i}\left(t_{1}, t_{2}\right)=\sum_{t=t_{1}}^{t_{2}} A R_{i, t}
$$

Em que, $t_{1}$ e $t_{2}$ são os períodos de acumulação dos retornos anormais que se localizam nos limites superior e inferior da janela do evento.

De acordo com Takamatsu, Lamounier e Colauto (2008), para analisar o efeito geral agregado do evento para todos os títulos individuais analisados, pode-se obter uma média dos retornos anormais acumulados dos títulos $\left[\overline{C A R}\left(t_{1}, t_{2}\right)\right]$. O seu cálculo para uma amostra com $N$ observações será dado pela seguinte equação: 
$\overline{C A R}\left(t_{1}, t_{2}\right)=\frac{1}{N} \sum_{i=1}^{N} \operatorname{CAR}_{i}\left(t_{1}, t_{2}\right)$

Diante do exposto pode-se estabelecer as seguintes hipóteses de pesquisa:

$\boldsymbol{H}_{\mathbf{0}}$ : $\mathrm{O}$ evento SEO não interfere no retorno das ações. Portanto, os retornos anormais são estatisticamente iguais a zero.

$\boldsymbol{H}_{\mathbf{1}}$ : O evento SEO interfere no retorno das ações. Portanto, os retornos anormais são estatisticamente significativos e diferentes de zero.

Para testar a hipótese nula utiliza-se a estatística de teste proposta por Campbell, Lo e MacKinlay (1997):

$$
J_{i}=\frac{\overline{C A R}_{t}\left(t_{1}, t_{2}\right)}{\sqrt{\bar{\sigma}^{2}\left(t_{1}, t_{2}\right)}} \approx N(0,1)
$$

Em que, $\sqrt{\bar{\sigma}^{2}\left(t_{1}, t_{2}\right)}$ é o desvio-padrão de $\overline{C A R}_{t}\left(t_{1}, t_{2}\right)$.

Em seguida, conforme Coelho (2008), os resultados obtidos referentes à estatística de teste permitirão analisar se houve, ou não, retornos anormais significativos nos pregões próximos ao evento. Para tanto, considera-se o nível de significância, $\alpha$, de $10 \%$ nesta pesquisa. Se o $p$-valor for menor que o nível de significância, rejeita-se a hipótese nula; caso contrário, se $p$-valor for maior ou igual ao nível de significância, não rejeita-se a hipótese nula.

\subsubsection{Procedimentos de Estimação e Testes de Pressupostos}

Esta seção apresenta os testes estatísticos necessários para a realização da pesquisa. $\mathrm{Na}$ execução das regressões estatísticas foram realizados os seguintes testes econométricos: (i) normalidade; (ii) linearidade; e, (iii) teste de independência dos resíduos. Foi utilizado o nível de significância estatística de $10 \%$ para todos os testes. Ressalta-se que para efeito desse estudo, todas as regressões foram estimadas utilizando-se as variâncias e erros-padrão consistentes para heterocedasticidade de White.

Para o teste de normalidade utilizou-se o teste de Kolmogorov-Smirnov (K-S). Segundo Maroco (2007, p. 134), o teste (K-S) "é usado para decidir se a distribuição da variável sob estudo $[\mathrm{F}(\mathrm{X})]$ numa determinada amostra provém de uma população com uma distribuição específica $F_{0}(\mathrm{X})$ ". Este teste é utilizado quando a média e o desvio padrão da população são conhecidos e procura-se verificar se a variável quantitativa segue uma distribuição normal padrão.

A linearidade foi verificada utilizando-se o coeficiente de correlação de Pearson. Gujarati (2006) destaca o coeficiente de correlação como sendo uma medida do grau de associação entre duas variáveis. Assim, caso o teste de linearidade apresentar $p$-valores maiores que o nível de significância de $10 \%$, rejeita-se a hipótese nula de associação entre as variáveis $R_{i, t}$ e $R_{m, t}$.

Para o teste de correlação serial dos resíduos das regressões estatísticas utilizou-se o teste- $d$ de Durbin-Watson (DW). De acordo com Gujarati (2006), este é o teste mais famoso para a detecção de correlação serial. Foram considerados, como valores de " $d$ " de cada série da amostra, intervalos entre $(0$ e 1,402) e $(2,598$ e 4) para rejeição de hipótese nula de ausência de 
autocorrelação, positiva ou negativa, excluindo da amostra as séries que porventura apresentarem valor crítico dentro desses intervalos.

Também, para complementar as análises, foi realizado o teste $t$ para diferença de médias. Este teste foi aplicado para testar a significância estatística das anormalidades dos retornos detectadas pós-evento. Realizou-se o teste entre as médias dos retornos anormais, comparandose os retornos anormais observados na amostra no período anterior e posterior à data do evento de registro da Oferta Pública Subsequentes de Ações. A metodologia utilizada para o teste baseou-se em pares de dias, sendo relacionado o primeiro dia anterior à data zero com o primeiro dia posterior à data zero $(-1 \mathrm{e}+1)$; o segundo dia anterior à data zero com o segundo dia posterior à data zero $(-2$ e +2$)$; e assim sucessivamente. De acordo com Takamatsu, Lamounier e Colauto (2008), para que a hipótese nula de igualdade de médias não seja rejeitada no teste $t$, o valor da estatística $t$ deverá estar situado entre os valores tabelados ao nível de significância adotado. Observou-se a necessidade da realização do teste de normalidade K-S para aferir o pressuposto de normalidade dos dados e posterior utilização do teste $t$.

As hipóteses estabelecidas para o teste $t$ foram:

$\boldsymbol{H}_{\mathbf{0}}$ : Os retornos anormais são estatisticamente iguais.

$\boldsymbol{H}_{\mathbf{1}}$ : Os retornos anormais são estatisticamente diferentes.

\section{APRESENTAÇÃO E ANÁLISE DOS RESULTADOS}

\subsection{Resultados dos testes de pressupostos estatísticos}

A suposição de que os retornos são normalmente distribuídos é necessária para a utilização do Modelo de Mercado e para os testes estatísticos do Estudo de Eventos. Para testar a normalidade, realizou-se o teste estatístico de Kolmogorov-Smirnov nas janelas de estimação, de eventos e de comparação. Considerando-se o nível de significância de 10\%, os resultados encontrados apontaram que não foram normalmente distribuídos: (1) os retornos para a janela de estimação do registro da SEO da Gafisa ( $p$-valor 0,000); (2) os retornos para a janela de eventos do registro da SEO do Banco Bradesco ( $p$-valor 0,025, Banco Bradesco 2); e, (3) os retornos para a janela de comparação da CSN ( $p$-valor 0,071$)$ e Gerdau ( $p$-valor 0,000 , Gerdau 2). Após os resultados apresentados para o teste de normalidade, foram excluídas as séries de dados das SEOs da Gafisa e do Banco Bradesco (Banco Bradesco 2), pois não atenderam ao pressuposto de normalidade nas janelas de estimação e de eventos. Já a séries de dados da SEOs da CSN e Gerdau (Gerdau 2) foram mantidas, nesta etapa, uma vez que tais séries atenderam ao pressuposto de normalidade nas janelas de estimação e eventos.

No teste de linearidade entre os retornos apresentados pelo Mercado (Ibovespa) e pelas ações das empresas da amostra, observou-se que apenas a série de dados da CSN teve seu $p$ valor $(0,164)$ superior ao nível de significância $10 \%$, não atendendo ao pressuposto de linearidade. Portanto, devido a isso, a série de dados da SEO da CSN foi excluída da amostra.

Quanto aos resultados do teste de autocorrelação dos resíduos, teste- $d$ de DurbinWatson (DW), verificou-se que oito séries apresentaram autocorrelação estatisticamente significativa: Braskem, Eletrobras 2, Gerdau 1, Perdigão 2, Sabesp 1, Vale do Rio Doce 1 e Vale do Rio Doce 2 apresentaram autocorrelação positiva; e, a Redecard apresentou autocorrelação negativa. As oito séries foram excluídas da amostra por não atenderem ao pressuposto de independência dos resíduos.

Após os resultados apresentados para os testes de pressupostos, a amostra final para as regressões pelo Modelo de Mercado consistiu de 27 séries compreendendo o evento SEO (Tabela 2). 
Nara Grossi Teixeira Guimarães - Oscar Neto de Almeida Bispo - Marcos Valério Martins Soares -

Vagner Antônio Marques

Tabela 2 - $\mathrm{N}^{0}$ de séries de eventos por ano (após testes de pressupostos estatísticos)

\begin{tabular}{cccccccccccc}
\hline Ano & $\mathbf{2 0 0 1}$ & $\mathbf{2 0 0 2}$ & $\mathbf{2 0 0 3}$ & $\mathbf{2 0 0 4}$ & $\mathbf{2 0 0 5}$ & $\mathbf{2 0 0 6}$ & $\mathbf{2 0 0 7}$ & $\mathbf{2 0 0 8}$ & $\mathbf{2 0 0 9}$ & $\mathbf{2 0 1 0}$ & Total \\
\hline Qtde. Eventos & 06 & - & - & 02 & 02 & 03 & 03 & 01 & 05 & 05 & $\mathbf{2 7}$ \\
\hline
\end{tabular}

Fonte: resultados da pesquisa

\subsection{Resultados relativos aos retornos anormais}

Seguindo os procedimentos metodológicos abordados na pesquisa, os retornos anormais foram calculados pelo Modelo de Mercado e os retornos observados pela forma logarítmica. Os parâmetros lineares $\alpha$ e $\beta$ foram estimados por meio de regressões conforme os Mínimos Quadrados Ordinários (MQO). Foram estimados os parâmetros para o cálculo dos retornos esperados e, em seguida, foram realizados os cálculos dos retornos anormais. Por conseguinte realizou-se a acumulação dos retornos anormais. Os resultados encontrados para $\alpha$ e $\beta$ são apresentados na Tabela 3.

Tabela 3 - Valores de $\alpha$ e $\beta$ para cada série de registro da emissão.

\begin{tabular}{|c|c|c|c|c|c|c|c|}
\hline Série & $\alpha$ & $p$-valor & $\beta$ & $p$-valor & $\mathbf{R}^{2}$ & Estat.-F & p-valor \\
\hline Ambev & 0.0008 & 0.598 & 0.4763 & $0.000^{*}$ & 0.238 & 11.624 & $0.002 *$ \\
\hline Bradesco 1 & -0.0050 & $0.010 *$ & 0.5368 & $0.000 *$ & 0.263 & 13.128 & $0.001 *$ \\
\hline Bco Brasil 1 & 0.0022 & 0.709 & 1.5056 & $0.000 *$ & 0.523 & 38.367 & $0.000 *$ \\
\hline Bco Brasil 2 & 0.0004 & 0.805 & 1.2295 & $0.000 *$ & 0.466 & 30.661 & $0.000 *$ \\
\hline Bco Brasil 3 & -0.0004 & 0.805 & 0.7912 & $0.000^{*}$ & 0.498 & 34.778 & $0.000 *$ \\
\hline Bradespar 1 & 0.0045 & 0.125 & 0.7678 & $0.000 *$ & 0.393 & 22.978 & $0.000 *$ \\
\hline Bradespar 2 & -0.0005 & 0.860 & 0.7529 & $0.007^{*}$ & 0.268 & 13.447 & $0.000 *$ \\
\hline Bradespar 3 & 0.0036 & 0.190 & 0.5660 & $0.031 *$ & 0.188 & 8.902 & $0.005 *$ \\
\hline CESP 1 & -0.0017 & 0.665 & 1.1434 & $0.000 *$ & 0.499 & 34.925 & $0.000 *$ \\
\hline CESP 2 & -0.0028 & 0.580 & 1.2936 & $0.000^{*}$ & 0.454 & 29.311 & $0.000 *$ \\
\hline Cyrela & -0.0058 & $0.093 *$ & 1.9118 & $0.000 *$ & 0.462 & 30.227 & $0.000 *$ \\
\hline Eletrobrás 1 & 0.0007 & 0.889 & 1.1422 & $0.000^{*}$ & 0.401 & 23.805 & $0.000 *$ \\
\hline Eletropaulo 1 & 0.0104 & $0.002 *$ & 0.8125 & $0.000^{*}$ & 0.413 & 24.969 & $0.000 *$ \\
\hline Embraer 1 & 0.0012 & 0.761 & 0.3657 & $0.068^{*}$ & 0.052 & 2.880 & $0.099 *$ \\
\hline Embraer 2 & -0.0023 & 0.346 & 0.5760 & $0.000 *$ & 0.209 & 9.979 & $0.003 *$ \\
\hline Gerdau 2 & 0.0038 & 0.218 & 1.2874 & $0.000 *$ & 0.670 & 69.934 & $0.000 *$ \\
\hline Gol & -0.0021 & 0.659 & 1.0412 & $0.000 *$ & 0.145 & 6.753 & $0.014 *$ \\
\hline JBS & -0.0032 & 0.489 & 1.2037 & $0.022 *$ & 0.130 & 6.093 & $0.019 *$ \\
\hline Light & 0.0049 & 0.121 & 0.3404 & $0.099 *$ & 0.067 & 3.464 & $0.072 *$ \\
\hline MMX & -0.0045 & 0.171 & 0.8781 & $0.003^{*}$ & 0.228 & 11.045 & $0.002 *$ \\
\hline Natura & 0.0016 & 0.475 & 0.5571 & $0.000 *$ & 0.306 & 15.993 & $0.000 *$ \\
\hline Perdigão 1 & 0.0006 & 0.851 & 1.5992 & $0.000 *$ & 0.500 & 35.069 & $0.000 *$ \\
\hline Petrobrás 1 & -0.0009 & 0.770 & 0.4366 & $0.016^{*}$ & 0.098 & 4.690 & $0.038 *$ \\
\hline Petrobras 2 & -0.0020 & 0.522 & 1.3861 & $0.000 *$ & 0.457 & 29.598 & $0.000 *$ \\
\hline Rossi Res. & 0.0013 & 0.680 & 2.8055 & $0.000 *$ & 0.626 & 57.922 & $0.000 *$ \\
\hline Sabesp 2 & -0.0029 & 0.138 & 1.0029 & $0.000 *$ & 0.410 & 24.637 & $0.000 *$ \\
\hline Usiminas & -0.0021 & 0.498 & 1.5834 & $0.000 *$ & 0.712 & 85.094 & $0.000 *$ \\
\hline
\end{tabular}

(*) Estatisticamente significativo a $10 \%$

Fonte:resultados da pesquisa.

Pode-se observar que todos os resultados foram significativos, tanto para os betas quanto para a estatística-F. Foi evidenciado que os resultados para $\mathrm{R}^{2}$ e estatística-F são consistentes com pesquisas anteriores [COELHO (2008); TAKAMATSU; LAMOUNIER; COLAUTO (2008); CASTRO e MARQUES (2012)] que utilizaram os mesmos procedimentos metodológicos. Dessa forma as inferências sobre os resultados foram realizadas considerando todas as emissões apresentadas na Tabela 3.

Quanto aos resultados dos cálculos dos retornos anormais médios $\left(\overline{A R}_{i t}\right)$ e retornos anormais médios acumulados $\left(\overline{C A R}_{i}\right)$ pode-se verificar a Tabela 4 . 
IMPACTO DO REGISTRO DE OFERTAS PÚBLICAS SUBSEQUENTES NOS RETORNOS DE AÇÕES DE EMPRESAS LISTADAS NO SEGMENTO

Tabela 4 - Resultados de $\overline{A R}_{i t}$ e $\overline{C A R}_{i}$ de cada série na janela de evento.

\begin{tabular}{lrrlrr}
\hline \multicolumn{1}{c}{ Série } & $\overline{\boldsymbol{A R}}_{\boldsymbol{i t}}$ & $\overline{\boldsymbol{C A R}}_{\boldsymbol{i}}$ & \multicolumn{1}{c}{ Série } & $\overline{\boldsymbol{A R}}_{\boldsymbol{i t}}$ & $\overline{\boldsymbol{C A R}}_{\boldsymbol{i}}$ \\
\hline Ambev & 0,00094 & 0,00231 & Embraer 2 & 0,01048 & 0,02116 \\
Banco Bradesco 1 & 0,00615 & 0,01447 & Gerdau 2 & $-0,00425$ & $-0,00797$ \\
Banco do Brasil 1 & $-0,01667$ & $-0,03219$ & Gol & $-0,00345$ & $-0,00401$ \\
Banco do Brasil 2 & 0,00325 & 0,01179 & JBS & 0,00441 & 0,01038 \\
Banco do Brasil 3 & 0,00228 & 0,00283 & Light & $-0,01881$ & $-0,03727$ \\
Bradespar 1 & 0,00092 & $-0,00008$ & MMX & 0,00030 & $-0,00438$ \\
Bradespar 2 & 0,00400 & 0,00556 & Natura & $-0,00261$ & $-0,00547$ \\
Bradespar 3 & 0,00523 & 0,01166 & Perdigão 1 & $-0,00832$ & $-0,01608$ \\
CESP 1 & $-0,00164$ & $-0,00547$ & Petrobrás 1 & $-0,00089$ & $-0,00010$ \\
CESP 2 & 0,00417 & 0,01173 & Petrobras 2 & 0,00118 & 0,00362 \\
Cyrela & 0,00251 & 0,00474 & Rossi Residencial & $-0,02274$ & $-0,04311$ \\
Eletrobrás 1 & $-0,00683$ & $-0,01842$ & Sabesp 2 & 0,00555 & 0,01021 \\
Eletropaulo 1 & $-0,02946$ & $-0,05968$ & Usiminas & 0,00328 & 0,00741 \\
Embraer 1 & $-0,00021$ & 0,00214 & & & \\
\hline
\end{tabular}

Fonte: resultados da pesquisa.

Os resultados encontrados na Tabela 4 mostram que aproximadamente $44,4 \%$ dos registros de SEOs resultaram em retornos anormais médios $\left(\overline{A R}_{i t}\right)$ negativos. Já os retornos anormais acumulados médios $\left(\overline{C A R}_{i}\right)$ apresentam aproximadamente $48,2 \%$ de resultados negativos. Se por um lado os resultados negativos apresentados na tabela pressupõem certa desvalorização nos retornos das ações, por outro lado observa-se que a maioria das séries da amostra projetaram uma valorização nos retornos dos títulos nos pregões próximos a data do registro na CVM. A Figura 1 apresenta os resultados obtidos dos retornos anormais médios por pregão da janela de eventos.

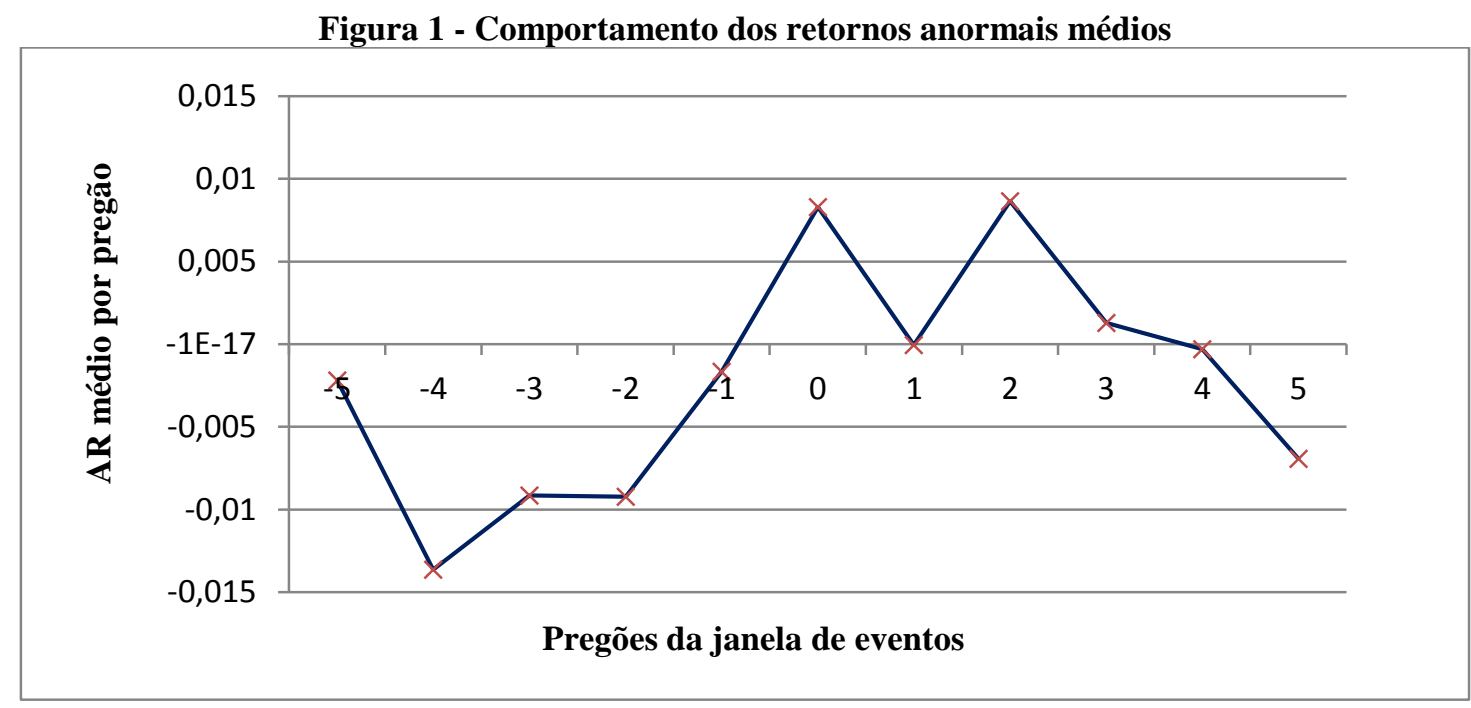

Fonte: resultados da pesquisa.

Conforme exposto na Figura 1, os pregões $t_{0}, t_{2}$ e $t_{3}$ apresentaram $\overline{A R}_{i t}$ positivos, em contrapartida os pregões $t_{-5}, t_{-4}, t_{-3}, t_{-2}, t_{-1}, t_{1}, t_{4}$ e $t_{5}$, apresentaram $\overline{A R}_{i t}$ negativos. Em seguida, como explicado anteriormente, a acumulação dos retornos foi realizada para avaliar a reação dos preços no mercado no decorrer da janela de eventos. A Figura 2 procura ilustrar o comportamento dos retornos anormais acumulados médios $\left(\overline{C A R}_{i}\right)$ das séries na janela de eventos. 
Nara Grossi Teixeira Guimarães - Oscar Neto de Almeida Bispo - Marcos Valério Martins Soares Vagner Antônio Marques

Figura 2 - Comportamento dos retornos anormais acumulados médios

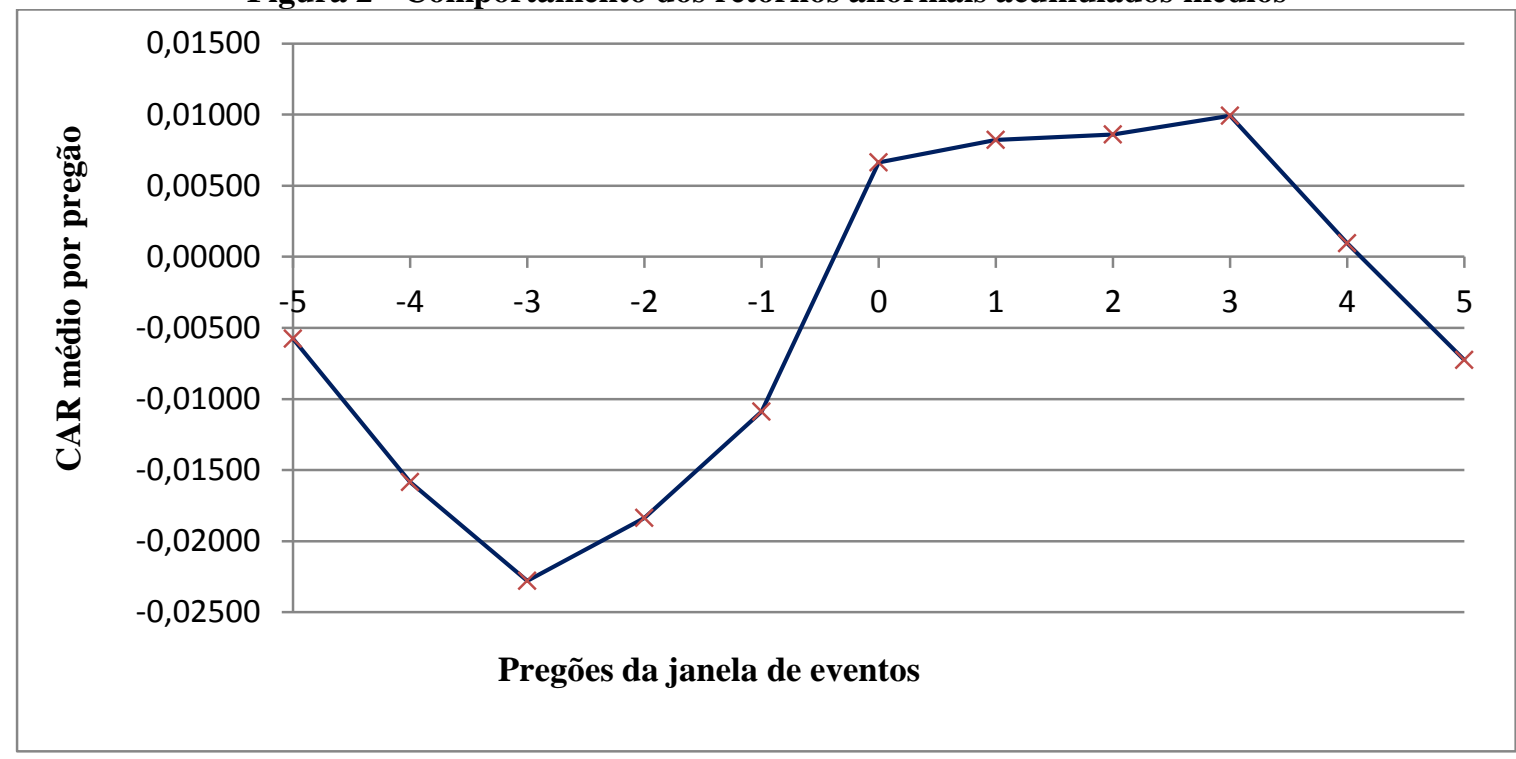

Fonte: resultados da pesquisa.

Observa-se que a partir do pregão $t_{-3}$ ao pregão $t_{1}$ os retornos anormais médios acumulados da janela de eventos começam a ter um discreto crescimento. Do pregão $t_{-1}$ ao pregão $t_{3}$ há um crescimento mais acentuado, indicando uma possível interpretação positiva do mercado em relação ao registro da emissão de novas ações na CVM. A Tabela 5 apresenta os resultados do teste de significância dos retornos anormais na janela de eventos, com base na estatística de teste $J_{l}$.

Tabela 5 - Resultados do estudo do registro na CVM na janela de eventos

\begin{tabular}{crrrrr}
\hline Pregões & $\overline{\boldsymbol{A R}}_{\boldsymbol{t}-\mathbf{5 , t + 5}}$ & $\overline{\boldsymbol{C A R}}_{\boldsymbol{t} \mathbf{- 5}, \boldsymbol{t}+\mathbf{5}}$ & $\boldsymbol{\sigma} \overline{\boldsymbol{C A R}}_{\boldsymbol{t}-\mathbf{5}, \boldsymbol{t}+\mathbf{5}}$ & \multicolumn{1}{c}{$\boldsymbol{J}_{\mathbf{1}}$} & \multicolumn{1}{c}{$\boldsymbol{p}$-valor } \\
\hline-5 & $-0,00220$ & $-0,00575$ & 0,02513 & $-0,22878$ & 0,818 \\
-4 & $-0,01363$ & $-0,01583$ & 0,03984 & $-0,51335$ & 0,610 \\
-3 & $-0,00915$ & $-0,02278$ & 0,04116 & $-0,55346$ & 0,582 \\
-2 & $-0,00923$ & $-0,01838$ & 0,03232 & $-0,56853$ & 0,569 \\
-1 & $-0,00166$ & $-0,01089$ & 0,03822 & $-0,28494$ & 0,772 \\
0 & 0,00829 & 0,00662 & 0,05095 & 0,13001 & 0,897 \\
1 & $-0,00006$ & 0,00823 & 0,04787 & 0,17190 & 0,865 \\
2 & 0,00865 & 0,00859 & 0,02995 & 0,28688 & 0,772 \\
3 & 0,00128 & 0,00993 & 0,02367 & 0,41953 & 0,674 \\
4 & $-0,00032$ & 0,00096 & 0,02477 & 0,03893 & 0,968 \\
5 & $-0,00693$ & $-0,00724$ & 0,03046 & $-0,23782$ & 0,810 \\
\hline
\end{tabular}

Fonte: resultados da pesquisa.

Conforme foi observado, não foram encontrados retornos anormais estatisticamente significativos na Tabela 5. Todos os p-valores encontrados são maiores que $10 \%$ (nível de significância máximo utilizado nesta pesquisa). Portanto, não se rejeita a hipótese nula de que os retornos anormais médios são iguais a zero. Pode-se concluir que o registro da emissão na CVM não é significativo nos retornos das ações das empresas.

Deve-se ressaltar que o mesmo teste foi realizado para verificar se ocorreram diferenças estatisticamente significativas no comportamento dos retornos anormais nos pregões subseqüentes à janela de eventos (janela de comparação, pregões +6 a +40 ). Foi observado que não ocorreram retornos anormais estatisticamente significativos na janela de comparação, confirmando a ausência de reflexos do evento nos retornos das ações das empresas que realizaram o registro de Ofertas Públicas Subsequientes de Ações. Os resultados apontaram p-valor acima do nível de significância de $10 \%$. Mesmo diante dos resultados apresentados, deve ser ressaltado que 
esta pesquisa limitou-se a verificar as implicações do registro de Ofertas Públicas Subseqüentes de Ações na CVM nos retornos das ações, não retratando as implicações de outros eventos corporativos ou de mercado que possam ter ocorrido no período analisado. Existe a possibilidade de ocorrência de outros eventos relevantes que trouxeram retornos anormais para o período. Entretanto, tais eventos não foram retratados nesta pesquisa.

Após a exposição dos resultados precedentes, realizou-se o teste $t$ para diferença de médias, visando testar a significância estatística das anormalidades dos retornos detectadas pósevento. Comparou-se os retornos anormais e anormais acumulados observados na amostra no período anterior e posterior à data do evento da deliberação em pares de dias. Considerando-se os valores calculados para a estatística $t$ e os respectivos valores críticos para a análise de rejeição ou não rejeição da hipótese nula de não existir diferenças entre as médias apresentadas. A seguir tem-se os resultados (Tabela 6):

Tabela 6 - Teste t para os retornos anormais e retornos anormais acumulados

\begin{tabular}{|c|c|c|c|c|c|}
\hline \multicolumn{3}{|c|}{ Retornos Anormais Diários } & \multicolumn{3}{|c|}{ Retornos Anormais Acumulados } \\
\hline Pregões & t calculado & t crítico & Pregões & t calculado & t crítico \\
\hline$(-1,+1)$ & $-0,2436$ & 2,0066 & $(-1,+1)$ & $-1,6218$ & 2,0086 \\
\hline$(-2,+2)$ & $-2,9392$ & 2,0066 & $(-2,+2)$ & $-3,1802$ & 2,0066 \\
\hline$(-3,+3)$ & $-1,6501$ & 2,0129 & $(-3,+3)$ & $-3,5797$ & 2,0181 \\
\hline$(-4,+4)$ & $-2,3063$ & 2,0066 & $(-4,+4)$ & $-2,2064$ & 2,0066 \\
\hline$(-5,+5)$ & 0,8776 & 2,0096 & $(-5,+5)$ & 0,1969 & 2,0086 \\
\hline
\end{tabular}

Fonte: resultados da pesquisa.

De acordo com os resultados apresentados na Tabela 6 os pares de retornos anormais dos pregões $(-1 ;+1),(-3 ;+3)$ e $(-5 ;+5)$, foram estatisticamente iguais, pois apresentaram estatística $t$ calculada menor que o $t$ crítico. Já os pares de retornos anormais dos pregões ($2 ;+2)$ e $(-4 ;+4)$ foram estatisticamente diferentes ( $t$ calculado maior que o $t$ crítico), apresentando. Ainda, conforme apresentado na Tabela 6 , as análises relacionadas aos retornos anormais acumulados evidenciaram que os pregões $(-1 ;+1)$ e $(-5 ;+5)$ foram estatisticamente iguais. No entanto, os retornos anormais acumulados referentes aos pregões $(-2 ;+2),(-3 ;+3)$ e $(-4 ;+4)$ foram estatisticamente diferentes após o registro na CVM.

Analisando-se os resultados de outras pesquisas observou-se que Medeiros e Matsumoto (2006) também estudaram, por meio da metodologia do estudo de evento, os retornos de ações relacionados a emissões públicas por empresas brasileiras listadas na BOVESPA, o estudo foi realizado entre os anos de 1992 e 2002 e buscava determinar como o mercado reagiu antes, durante e depois do anúncio da emissão. O estudo utilizou uma amostra de 80 emissões de empresas e os resultados demonstraram que ocorrem retornos anormais negativos na data do anúncio.

Iquiapaza et. al (2009), utilizou o estudo de evento para analisar a reação do mercado ao anuncio de duas formas de lançamento público primário não inicial de ações: ofertas amplas e ofertas restritas, para empresas listadas no segmento Bovespa. Observou-se o desempenho dos preços antes, durante e até um ano após a data de anúncio de emissões realizadas entre 1995 e 2005. Os autores constataram que pelo modelo de retornos ajustado ao mercado os resultados se mostraram não significativos para as duas formas de emissões (com exceção para os retornos negativos no longo prazo das ofertas amplas). Já no modelo de retornos ajustado ao risco e ao mercado, os resultados para ofertas amplas mostraram que ocorrem retornos anormais negativos na data do anuncio, e que apresentam retornos negativos ao longo de um ano.

Os resultados encontrados nesta pesquisa não vão de acordo com aqueles encontrados por Medeiros e Matsumoto (2006) e Iquiapaza et al. (2009), uma vez que não foram encontrados retornos anormais estatisticamente significativos na data do registro da emissão subsequente, conforme resultados da estatística $J_{i}$. A inobservância de retornos anormais nos pregões $t_{0}$ e $t_{1}$ 
Nara Grossi Teixeira Guimarães - Oscar Neto de Almeida Bispo - Marcos Valério Martins Soares Vagner Antônio Marques

reforça a hipótese de eficiência semiforte no contexto brasileiro, na medida em que os preços se ajustam em períodos próximos ao evento e não imediatamente à divulgação do mesmo. Por outro lado, a utilização da data de registro na CVM pode ser uma das explicações de tal fato, pois em meses anteriores ao registro naquele órgão, um evento deliberativo sobre a oferta pública é divulgada ao mercado, diante disso, o registro na CVM apenas homologa uma decisão anteriormente feita e divulgada ao mercado.

Segundo Fama (1970), estudos sobre os reflexos de determinados eventos nos preços das ações podem trazer suporte de evidência para o modelo de eficiência de mercado. Camargos e Barbosa (2003b) destacam que os preços dos títulos das empresas negociados no mercado de capitais são influenciados por eventos ou informações relevantes que provocam alterações em suas cotações.

Damodaran (2004) ressalta que se o mercado reage imediatamente à divulgação de uma informação relevante, a hipótese de eficiência semiforte do mercado é confirmada. Em contrapartida, se a informação divulgada oscila entre aumentos e reduções graduais nos preços, há indícios de ineficiência semiforte do mercado. Os retornos anormais observados na janela de estimação e comparação desta pesquisa apresentam um ajuste lento e gradual dos preços das ações às informações divulgadas pelo registro na CVM, apontando para a ineficiência semiforte do mercado. Se, por outro lado, o conteúdo informacional fosse totalmente absorvido nos preços das ações, não seria possível obter retornos acima do esperado ao longo do tempo, evidenciando a eficiência do mercado.

\section{CONSIDERAÇÕES FINAIS}

Entre as alternativas mais eficientes de financiamento das operações de uma empresa, encontra-se a captação de recursos via emissão de novas ações para o levantamento do capital necessário ao desenvolvimento e viabilização dos projetos de investimento pretendidos. No decorrer das atividades das empresas sempre haverá oportunidades de crescimento, e a necessidade de capital externo poderá ocasionar o retorno ao mercado, acarretando na aquisição de novos sócios e consequentemente novo financiamento para suas atividades.

A colocação e emissão de novas ações no mercado por meio de uma Seasoned Equity Offering - SEO é uma forma vantajosa que as empresas de capital aberto dispõem para levantar recursos. Neste sentido, o objetivo da presente pesquisa foi analisar os impactos das ofertas públicas subsequentes nos retornos de ações de empresas listadas no segmento Bovespa e que fizeram parte do Ibovespa no mesmo período.

Para tanto, foram coletadas na CVM as datas de registro da emissão de novas ações, além das cotações das ações dessas empresas em datas próximas aos eventos. Os dados analisados foram referentes a 27 empresas entre os anos de 2001 a 2010. Foram estimadas regressões a partir do Ibovespa e dos retornos observados das ações na janela de estimação. $\mathrm{O}$ modelo de determinação foi o modelo do retorno ajustado ao risco e ao mercado, atendendo-se ao pressuposto de normalidade, linearidade e autocorrelação dos resíduos, premissas básicas para utilização do modelo.

Como resultados, não foram encontrados retornos anormais estatisticamente significativos, haja vista que todos os p-valores são maiores que o nível de significância máximo utilizado na pesquisa. Portanto, não se rejeitou a hipótese nula de que os retornos anormais médios são iguais a zero. Diante disso, conclui-se que o registro da emissão na CVM não é significativo nos retornos das ações das empresas. Os resultados também apontaram que não ocorreram retornos anormais estatisticamente significativos na janela de comparação, confirmando a ausência de reflexos do evento nos retornos das ações das empresas que realizaram o registro de Ofertas Públicas Subsequentes de Ações. 
Como limitações do estudo, esta pesquisa verificou as implicações do registro de Ofertas Públicas Subsequentes de Ações na CVM nos retornos das ações, não retratando as implicações de outros eventos corporativos ou de mercado que possam ter ocorrido no período analisado.

Para futuras pesquisas, sugere-se a aplicação da metodologia aqui adotada a outros eventos, pesquisas que visam analisar as reações do mercado de capitais frente às emissões de ações, mais especificamente as ofertas públicas subsequentes. Estudos desta natureza podem contribuir para uma melhor percepção da relevância das informações contábil-financeiras para os agentes nos mercados de capitais. O retorno e outros reflexos advindos desse tipo de oferta podem provocar impactos substanciais para o mercado acionário. Um estudo de evento da emissão se SEO's aprofundado em termos do tamanho da amostra, utilizando-se métodos estatísticos e ferramentas mais precisas para tratamento dos dados, as quais permitam a verificação dos níveis de interdependência das variáveis envolvidas na explicação dos cálculos de beta e de retorno histórico, seria de grande relevância no mercado de capitais.

\section{REFERÊNCIAS}

ASSAF NETO, Alexandre. Mercado financeiro. 7. ed. São Paulo: Atlas, 2007.

BERK, Jonathan; DEMARZO, Peter. Finanças empresariais. Bookman, 2009.

BISPO, Oscar de Almeida. Gerenciamento de resultados contábeis e o desempenho das ofertas públicas subsequentes de ações de empresas brasileiras. 2010. $141 \mathrm{f}$. Tese (Mestrado em Contabilidade e Controladoria) - Faculdade de Ciências Econômicas, Universidade Federal de Minas Gerais, Belo Horizonte, 2010.

BMF\&BOVESPA - Bolsa de mercadorias 7 futuros de São Paulo. Folhetos Educativos. Introdução ao mercado de capitais. 2010. Disponível em: <http://www.bmfbovespa.com.br/ptbr/educacional/download/merccap.pdf >. Acesso em: 22 ago. 2011.

BRESSAN, Valeria Gama Fully et al. Rentabilidade e assimetria de informação em empresas selecionadas na Bovespa. Rev. Ciênc. Admin., Fortaleza, v. 13, n. 2, p. 223-233, nov. 2007.

BRIGHAN, Eugene F.; EHRHARDT, Michael C. Administração financeira: teoria e prática. São Paulo: Pioneira Thomson Learning, 2006.

CAMARGOS, Marcos Antonio; BARBOSA, Francisco Vidal. Teoria e evidência da eficiência informacional do mercado de capitais brasileiro. Caderno de pesquisas em administração, São Paulo, v. 10, n. 1, p. 41-55, jan./mar. 2003a.

CAMARGOS, Marcos Antonio; BARBOSA, Francisco Vidal. Estudos de evento: teoria e operacionalização. Caderno de pesquisas em administração, São Paulo, v. 10, n. 3, p. 01-20, jul./set. $2003 b$.

CAMPBELL, J. Y.; LO, A. W.; MACKINLAY, A. C. The econometrics of financial markets. 2. ed. New Jersey: Princeton University Press, 1997. 611 p.

CASTRO, Rafaela A. D.; MARQUES, V.A. A influência da publicação das demonstrações contábeis no mercado de capitais. Revista Enfoque Reflexão Contábil, Maringá, v. 32, n. 1, p. 109-124, jan./abr. 2012. doi:10.4025/enfoque.v32i1.15362

COELHO, Renata D. P. A emissão de debêntures e seus reflexos sobre o retorno e o risco das ações de empresas brasileiras. 2008. Dissertação (Mestrado) - Centro de Pesquisa e PósGraduação em Administração, Universidade Federal de Minas Gerais. Belo Horizonte, 2008, $212 \mathrm{f}$.

DAMODARAN, Aswath. Gestão estratégica do risco: Uma referência para a tomada de riscos empresariais. Porto Alegre: Bookman, 2008. 
Nara Grossi Teixeira Guimarães - Oscar Neto de Almeida Bispo - Marcos Valério Martins Soares Vagner Antônio Marques

DENIS, D. J.; SARIN, A. Is the Market Surprised by Poor Earnings Realizations Following Seasoned Equity Offerings? The Journal of Financial and Quantitative Analysis, v. 36, n. 2, Special Issue on International Corporate Governance, pp. 169-193, Jun., 2001,

DIAS, Edeson M. Decisões de financiamento de empresas brasileiras de capital aberto maduras: testando peckingorder. 2007. 64 f. Dissertação (Mestrado) - Programa de PósGraduação e Pesquisa em Administração e Economia, Ibmec, Rio de Janeiro, 2007.

FAMA, E. F. Efficient capital markets: a review of theory and empirical work. The Journal of Finance, Chicago, v. 25, n. 2, p. 383-417, Mai. 1970. Doi: 10.2307/2325486

FAMA, E. F. Efficient capital markets II. The Journal of Finance, Chicago, v. 46, n. 5, p. 1575-1617, Dez. 1991. Doi: 10.1111/j.1540-6261.1991.tb04636.x

FERREIRA, Robson Diego. Análise de risco e retorno das ações de empresas listadas no nível 1 de governança corporativa em relação ao mercado tradicional da bolsa de valores de São Paulo. 2008. 95f. Trabalho de Conclusão de Curso em administração, Universidade Federal do Rio Grande do Sul, Porto Alegre, 2008.

GIL, Antônio C. Métodos e técnicas de pesquisa social. 5. ed., São Paulo: Atlas, 2007.

GUJARATI, Damodar N. Econometria básica. 4 ed. Rio de Janeiro: Elsevier, 2006.

HENDRIKSEN, Eldon S.; VAN BREDA, Michael F. Teoria da contabilidade. 1. ed. São Paulo: Atlas, 1999.

IQUIAPAZA, Robert Aldo et al. Informação dos insiders e seu efeito sobre os preços em duas formas de emissão de ações na Bovespa. Revista Contabilidade Vista \& Revista, v. 20, n. 2, p. 15-37, abr./jun. 2009.

LOUGHRAN, T.; RITTER, J. R. The Operating Performance of Firms Conducting Seasoned Equity Offerings. The Journal of Finance, v. 52, n. 5, p. 1823-1850, Dec., 1997. Doi: 10.1111/j.1540-6261.1997.tb02743.x

MAROCO, Joao. Análise estatística: com utilização do SPSS. 3. ed. Lisboa: Sílabo, 2007.

MEDEIROS, Otavio Ribeiro; MATSUMOTO, Alberto Shigueru. Emissões públicas de ações, volatilidade e insider information na Bovespa. Revista Cont. Fin., n. 40, p. 25-36, jan./abr. 2006. http://dx.doi.org/10.1590/S1519-70772006000100003

MYERS, Stewart C. The Capital Structure Puzzle. The Journal of Finance, v. 39, n. 3, Papers and Proceedings, Forty-Second Annual Meeting, American Finance Association, San Francisco, CA, December 28-30, 1983. p. 575-592, Jul., 1984. Doi: 10.1111/j.15406261.1984.tb03646.x

RANGAN, S. Earnings management and the performance of seasoned equity offerings. Journal of Financial Economics. v. 50, p. 101-122, 1998. http://dx.doi.org/10.1016/S0304405X(98)00033-6

SANTOS, Luiz et al. Teoria da contabilidade. São Paulo: Atlas, 2007.

TAKAMATSU, Renata Turola; LAMOUNIER, Wagner Moura; COLAUTO, Romualdo Douglas. Impactos da divulgação de prejuízos nos retornos de ações de companhias participantes do Ibovespa. Revista Universo Contábil, v.4, n.1, p. 46-63, jan./mar. 2008.

TEOH, S. H.; WONG, T. J.; RAO, G. R. Are accruals during Initial Public Offerings opportunistic? Review of Accounting Studies, v. 3, pp. 175-208, 1998. Doi:10.1023/A:1009688619882 